\title{
Détermination des dimensions caractéristiques d'un réservoir d'air sur une installation élévatoire
}

\section{Determining the dimensions \\ of an air reservoir to be used on a pumping line}

CH. DUBIN

INGENIRUR DES ARTS ET MANUFACTIRES.
PAR

ET

\author{
A. GUENEAT \\ ANGIEN ÉLĖVE DE L'ÉCOLE POLYTECHNIQUE, \\ INGÉNIEUR GIVIL DES PONTS ET CHAUSSÉES
}

\begin{abstract}
Dans cet article, les autenrs exposent le résultat de lenrs recherches sur la determination des dimensions d'un réservoir d'air en appliquant la therie du coup de belier dondes. Cette étude nécessitait, pour y voit clair, l'exécution d'un grand nombre d'bures Bergeron. Les auteurs ont wis au point pour cela une méthode qui permet anx mochines à cartes perfores de faire ce travail.

II est a noter que depmis la rédaction de cet article, de nouvelles machines (I.B.M. type 650) ont apparu en France. Les aufeurs estiment que l'épure de la fipure 12 qui, il $y$ a six mois. demandait une demi-heure, potarraif ètre achevéc anjonthmi n... frois minutes.
\end{abstract}

\begin{abstract}
In this paper the authors state the results they have obtained concerning the determination of the size of an air vessel with the use of the theory of surges. The graphical method as applied by Mr. Bergeron required an important number of graphs to obtain a clear view of the process.

The authors have succeeded in establishing a method which allows the electronic computers with perforated cards to solve the problem.

Since this article was written a new computer (I.B.M. model 650) is available in France.

It may be estimated that the graph Fig. 12 which required half an hour six months ago could rom be established in... three minutes.
\end{abstract}

La genéralisation de l'emploi de l'énergie "lestrique, dans les usines élévatoires de distribution d'eau, a posé, avec une acuité inconnue jusqu"iei, la question des coups de bélier.

Dans les anciennes installations, pourvaes de moteurs à vapeur on de moteurs à combustion interne, on nobservait pratiquement jamais de coup de bélier consécutif à un arrèt de la pompe.

En effet, les moteurs comportaient toujours un rolant assez important qui, par son inertie, empechait tout arrèt brusque. Et, d'autro part, le Chef d'Usine restait maitre des opérations d'arrèt et pouvait les conduire de façon à minimiser le coup de bélier.

Aujourd'hui, par contre, il devient indispensable de protéger les installations contre les coups de bélier, car les groupes électro-ponıpes modernes n'ont qu'une très faible inertie, et, en outre, l'arrèt du courant peut se produire brusquement sans qu'aucune précaution ait pu etre prise pour en temporiser l'effet.

Or, les conditions de fonctionnement des grou- 
pes (hateur-vitesse) sont telles que la protection par réservoir d'air s'impose le plus souvent.

Ce réservoir, on le sait, a deux effets diff'érents :

Pendant la première période (l'eau continuant à s'écouler dans le sens pompe-réservoir d'extrẻInité, avec une vitesse positive), le réservoir d'air, en se détendant, fournit de l'eau à la canalisation ef tempère la baisse de pression qui se produirait dans une installation non protégée.

Pendant la deuxième période, le sens du courant s'est inversé et le réservoir d'air reçoit de l'eat. La pression, sans atteindre la valeur de la surpression observée sur une conduite non prolégée, peut néanmoins croître au-dessus de la charge limite qu'est capable de supporter l'installation.

Pour diminuer cette surpression, le remede est connu : entre la canalisation et le réservoir d'air, on place un organe (ajutage de Borda ou clapet troué) qui crée une perte de charge dissymétrique et qui présente en outre l'avantage d'amortir rapidement les oscillations de la pression autour de la charge statique; ces oscillations, en effet, peuvent se poursuivre assez longtemps, notamment dans les installations où la perte de charge dans la canalisation est trop faible pour créer, à elle seule, un aniortissement appréciable (*).

L'ensemble réservoir d'air-clapet troué agit, en quelque sorte, comme l'ensemble ressortamortisseur d'une voiture : si la roue doit passer sur une bosse, le ressort l'oblige à épouser le profil de cette bosse et l'amortisseur empêche le retour brusque du ressort et les nombreuses oscillalions consécutives.

Qualitalivement, l'allure dù phénomène est bien connue. L'étude en est facile grâce à la méthode Bergeron, mais néccssite une épure dans chaque cas particulier.

De nombreuses études ont été poursuivies pour permettre d'obtenir une solution plus rapide du problème, notamment par MM. SLiosbera, VIBErT, Combes et Borot qui ont publié des abaques permettant de déterminer les pressions maximum et minimum dans le réservoir, en fonction du volume du réservoir et de la perte de charge introduite par le clapet.

Ces abaques ont ćté établis à partir de l'hypothèse simplificatrice de l'oscillation en masse. II est exact que, dans la plupart des cas, cette hypothèse donne une valeur de la pression minimum, au droit du réservoir, très pen différente de la valeur donnée par la théorie plus précise du coup de bélier d'ondes.

Mais dans la recherche de la pression mini-

(*) Dans la suite de l'exposé, nous supposerons cette perte de charge dans la conduite négligeable, ce qui conslituc le cas le plus défavorable. Nous laissons au lecteur le soin d'extrapoler dans le cas où elle ne le seraít pas. mum, en un point quelconque de la conduite, la théorie de l'oscillation en masse donne une loi linéaire de variation en fonction de la distance; or, ceci est faux, car, en réalité, la courbe des pressions minima peut présenter une concavité assez accentuée.

Le réservoir peut et doit éviter tout risque de cavitation en tout point de la canalisation. Et nous savons que, pour décider si oui ou non il y aura cavitation en un point quelconque de la conduite, il faut superposer la courbe des pressions minima et le profil en long de la conduite dessinés à la même échelle.

La sécurité exige que le profil en long soit entièrement au-dessous de la courbe des pressions minima ou du moins n'empiète pas sur elle d'une hauteur supérieure à 5 ou $6 \mathrm{~m}$, au-delà de laquelle le siphonnage pourrait ne plus avoir lieu et la cavitation apparaitrait.

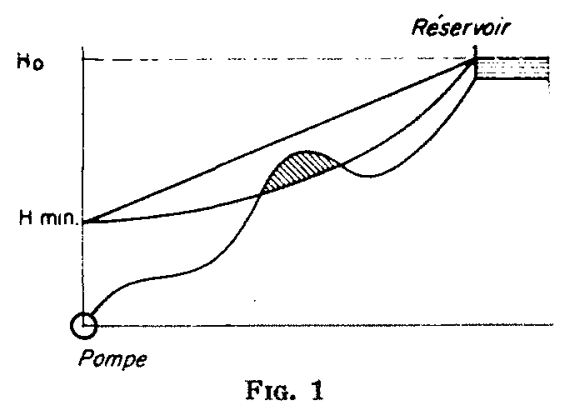

Or, si nous comparons les deux courbes (fig. 1), nous voyons que, dans la zone hachmrée, le danger de cavitation existe (contrairement à ce qu'indique la théorie de l'oscillation en masse représentée ici par la droite joignant le point $\mathrm{H}_{\text {min }}$ au réservoir).

C'est pourquoi l'emploi de ces abaques doit être strictement limité aux profils en long présentant une forte concavité (courbe $A$ de la

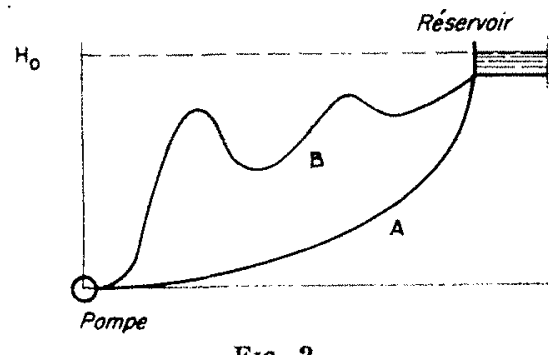

FiG. 2

fig. 2); il doit être proscrit pour des profils en long accidentés, du type de la courbe $B$.

Pour faciliter la détermination du volume du réservoir d'air, en vue de la protection complète de l'installation, nous avons étudié l'allure gé- 
nérale de ces courbes de pressions minima et nous donnons plus loin les résultats de cette étude.

Après avoir examiné ce qui se passe dans la première partie du phénomène (domaine des vitesses positives), voyons maintenant ce qui se passe dans la seconde partie, après l'inversion du courant, en supposant que le dispositif amortisseur soit un clapet percé.

Lorsqu'il n'y a pas de clapet du tout, la pression remonte dans la conduite et le réservoir, à une valeur $H_{\max }$ telle que :

$$
\mathbf{H}_{\text {max }}-\mathbf{H}_{0}>\mathbf{H}_{0}-\mathbf{H}_{\text {min }}
$$

$\mathrm{H}_{0}$ représentant la charge statique (voir fig. 3).

Lorsqu'il existe un clapet plein, la fermeture de ce clapet stabilise a $H_{\text {min }}$ la pression dans le

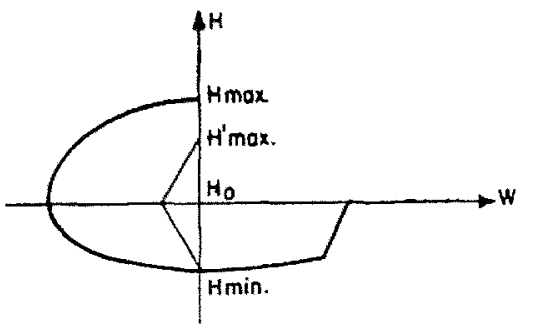

FIG. 9

réservoir et crée, dans la conduite, une surpression $\mathrm{H}_{\max }^{\prime}<\mathrm{H}_{\min }$ telle que :

$$
\mathbf{H}_{\text {inas }}^{\prime}-\mathbf{H}_{0}=\mathrm{H}_{0}-\mathrm{H}_{\text {min }}
$$

Enfin, si l'on perce ce clapet d'un trou, on observera dans la conduite une surpression $\mathrm{H}^{\prime \prime}{ }_{\max }$ qui sera, pour des dimensions suffisamment petites de l'orifice, inférieure à $\mathrm{H}_{\text {max }}$.

On concoit donc qu'il y ait, pour le clapet, un ćtranglement optimum pour lequel la surpression dans la conduite sera minimum, et nous pensons que, pour un réservoir, comme pour une cheminée d'ailleurs, il n'y a pas d'autre définition de l'étranglement optimum.

Or, cet optimum, la théorie de l'oscillation en masse ne peut le déterminer. En effet, même st loscillation en masse est netfement établie en fin de la première période, la fermeture du clapet va modifier lallure du phénomène et crécr un nouveau coup de bélier d'ondes.

Les hydrauliciens qui n'ont pas l'hahitude des épures Bergeron peuvent se demander par quel mystère un calculateur peut déterminer s'il a affaire à un coup de bélier d'ondes ou à une oscillation en masse. C'est extrêmement simple : il suffit de joindre par une courbe les points représentatifs des états (hauteur, vitesse) en un même instant pour différents points de la conduite (fig. 4).

Si cette courbe est une verticale, on peut dire qu'il y a oscillation en masse. Par contre, si cette courbe est quelconque (et en réalité on sait qu'il peut s'en présenter toute une gamme avec

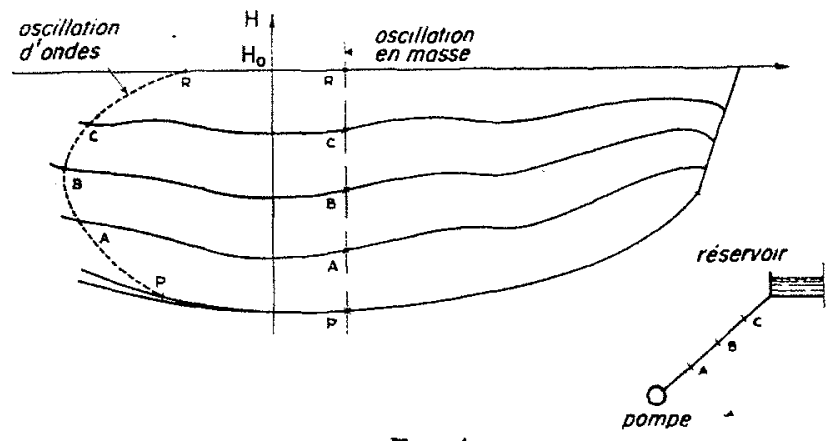

FIG. 4

points de rebroussement, boucles, etc.), il n'y a pas oscillation en masse.

Dans le cas qui nous intéresse, au début de l'arrêt bruscrue il n'y a pas oscillation en masse, mais elle s'établit assez rapidement (du moins pour un réservoir d'air assez grand) et c'est pour cette raison que les résultats donnés par les abađues sont assez voisins des résultats réels

Mais, dès que le clapet trouć se referme, un nouveau coup de bélier d'ondes apparait, d'autant plus accentué que l'orifice est plus étroit. La théorie de l'oscillation en masse ne peut plus nous renseigner sur l'allure du phénomène et encore moins nous indiquer les dimensions optima de l'étranglement. Dès lors, il n'y a plus qu'une solution si l'on veut déterminer ces dimensions : faire de nombreuses épures.

C'est une solution pour le moins fastidieuse, exigeant une grande précision graphique, et tous ceux qui ont travaillé ce genre d'épures savent combien, dans la zone des vitesses négatives, les points successifs ont tendance à s'emmêler jusqu'à rendre l'épure inextricable.

Heureusement, il est, depuis ruelques années. un instrument qui peut faire, sans se lasser, des centaines et des centaines d'épures et qui peut, sans se tromper, donner les coordonnées des points successifs à la précision relative de $10^{-7}$ évidemment plus que surabondante.

Cet instrument, c'est la machine à calculer à cartes perforées. Le laboratoire de calcul scientifique de la Société I.B.M. a bien voulu accepter de faire exécuter une suite d'épures Bergeron par ses machines, et c'est grâce à cela que nous pouvons donner une solution au problème de l'étranglement ovtimum du clapet amortisseur d'un réservoir d'air. 


\section{PREMIERE PARTIE}

\section{EXPOSE DE LA METHODE DE CALCUL}

\section{NOTATIONS}

$\mathrm{H}_{0}$ : pression statique absolue;

$\mathrm{H}_{\sigma}$ : pression absolue dans la conduite à l'instant $t$;

$\mathrm{H}_{\mathrm{R}}$ : pression absolue dans le réservoir à l'instant $t$;

$w_{0}$ : vitesse initiale en régime permanent;

$w$ : vitesse à l'instant $t$;

$a$ : célérité des ondes dans la conduite;

$\mathrm{L}$ : longueur de la conduite;

$\sigma$ : section de la conduite;

$v_{e}=\mathrm{L} \sigma=$ volume de la conduite;

$v_{0}$ : volume de l'air contenu dans le réservoir sous la pression $\mathrm{H}_{0}$;

$v$ : le même volume sous une pression $\mathrm{H}_{\mathrm{R}}$;

$\alpha w w^{2}$ : perte de charge due au clapet dans le sens conduite-réservoir d'air.

En outre, pour avoir des équations homogènes, nous ferons à chaque instant intervenir les deux quantités :

$$
\begin{aligned}
& \mathrm{A}=\frac{a w_{0}}{g \mathrm{H}_{0}} \\
& \mathrm{~B}=\frac{w_{0}^{2}}{g \mathrm{H}_{0}} \cdot \frac{v_{c}}{v_{0}}
\end{aligned}
$$

(la quantité $B$ est le double de la quantité $n$ qui intervient dans l'abaque de MM. Combes et Borot).

\section{Position du problème.}

Les équations du problème sont immédiatement déduites de la construction d'une épure Bergeron dans laquelle la perte de charge de la conduite est négligée.

Tout d'abord, la différence entre la charge dans la conduite et la charge dans le réservoir d'air est égale à la perte de charge du clapet :

$$
\mathrm{H}_{c}-\mathrm{H}_{\mathrm{Il}}=\alpha w^{2}
$$

lorsque l'eau circule dans le sens conduite-réservoir (clapet fermé); cette différence est nulle lors que l'eau circule dans l'autre sens (clapet ouvert).

La deuxième équation est obtenue en écrivant qu'un observateur met le temps :

$$
\tau=\frac{2 \mathrm{~L}}{a}
$$

pour faire un aller et retour dans la conduite en se déplaçant sur des droites Bergeron de pentes $\pm a / g$ (dans un diagramme charge-vitesse) :

$$
\left(\mathrm{H}_{c}-\frac{a}{g} w\right)_{t}=2 \mathrm{H}_{0}-\left(\mathrm{H}_{c}+\frac{a}{g} w\right)_{t-\tau}
$$

La troisième équation exprime la loi de variation du volume de l'air contenu dans le réservoir avec $H v=$ constante pour la loi isotherme.

En fait, l'équation (2) n'étant pas susceptible d'une intégration mathématique, nous opérerons, non pas sur des différentielles, mais sur des différences finies. Si donc la période $\tau$ est fractionnée en $\mathbf{N}$ parties égales, la loi caractérisant le réservoir d'air s'écrira :

$$
v_{t}-v_{t-(\tau / \mathrm{N})}=\sigma \cdot \frac{w_{t}+w_{t-(\tau / N)}}{2} \cdot \frac{\tau}{N}
$$

ou encore :

$$
\begin{aligned}
\frac{1}{\mathrm{H}_{\mathrm{R}, t}}- & \frac{1}{\mathrm{H}_{\mathrm{R}, t-(\tau / \mathrm{N})}} \\
& =\frac{\sigma}{\mathrm{H}_{0} v_{0}} \cdot \frac{w_{t}+w_{t-(\tau / \mathrm{N})}}{2} \cdot \frac{\tau}{\mathrm{N}}
\end{aligned}
$$

Les trois équations (1), (2), (3) permettent, à tout instant, de déterminer la vitesse $w$, la charge $\mathbf{H}_{c}$ dans la conduite et la charge $\mathrm{H}_{\mathrm{R}}$ dans le réservoir, en fonction de valeurs de ces mêmes variables aux instants antérieurs.

\section{Modification de ces équations.}

La construction des épures par le calcul ayant été demandée à des machines électroniques, il nous fallait établir une programmation simple 
de travail; ceci nous a conduits à changer d'inconnues en les remplacant par des quantités proportionnelles, les coefficients de proportionnalité ayant été déterminćs de façon à obtenir le syslime suivant d'équations réduites :

$$
\left\{\begin{array}{l}
z_{1} \cdots y_{1}=\lambda x_{1}^{2} \\
\left(z \cdots x_{1}=2 z_{1}-(z+x)_{1-z}\right. \\
\frac{1}{y_{r}}-\frac{1}{y_{t}-x_{1}}=x_{1}+x_{1-1-N)}
\end{array}\right.
$$

$x, y, z$ etant liés aux inconnues $w, \mathrm{H}_{\mathrm{R}}, \mathrm{H}_{v}$ par les relations :

$$
x=\frac{w}{w_{0}} \cdot x_{0} \quad y=\frac{\mathrm{H}_{\mathbf{R}}}{\ddot{\mathrm{H}}_{i}} \cdot y_{0} \quad z=\frac{\mathrm{H}_{i}}{\mathrm{H}_{i}} \quad z_{0}
$$

avec :

$$
x_{t 1}=\sqrt{\frac{\mathrm{B}}{\mathrm{N}}} \quad y_{0}=z_{0}=\sqrt{\frac{\mathrm{B}}{\mathrm{A}^{2} \mathrm{~N}}}
$$

et :

$$
\lambda=\frac{x w_{0}^{\prime \prime}}{\mathrm{H}_{4}} \sqrt{\frac{-N}{\mathrm{~A}^{2} \mathrm{~B}}}
$$

Enfin, pour simplifier à l'extrême, nous avons également introduit les quantités suivantes :

$$
\begin{aligned}
& \mathrm{S}_{t}=2 z_{0}-(z+x)_{t} \\
& \mathrm{~T}_{t}=x_{t}+\frac{1}{y_{t}}
\end{aligned}
$$

ce qui, finalement, nous donne le système suivant à résoudre :

$$
\left\{\begin{array}{l}
z_{t}-y_{t}=\lambda x_{t}^{2} \\
z_{t}-x_{t}=\mathrm{S}_{t-\tau} \\
\frac{1}{y_{t}}-x_{t}=\mathrm{T}_{t-(\pi / N)}
\end{array}\right.
$$

\section{Résolution pratique des équations.}

Pagmiar cas : $x>\|$ et $t<\tau$

Pendant cette première période, les droites Bergeron (équations 2 ou 12) sont toutes confondures; le système d'équations se rẻduit donc à :

$$
\left\{\begin{array}{l}
z_{t}=y_{t} \\
z_{t}-x_{t}=z_{b}-x_{t} \\
\frac{1}{y_{t}}-x_{t}=\mathrm{T}_{t-i \mathrm{~s}}
\end{array}\right.
$$

dont la solution est immediate

$$
\begin{aligned}
& z_{1}=-\frac{\mathrm{T}_{t-(\tau / N)}-\left(z_{0}-x_{0}\right)}{2} \\
& +\sqrt{+} \sqrt{\left[\frac{T_{t-(\tau / N)}-\left(z_{0}-x_{0}\right)}{2}\right]^{-\cdots}+1}
\end{aligned}
$$

$x_{1}=z_{t}-\left(z_{0}-x_{0}\right)$

DEUXIÈME CAS : $x>0$ et $t>\tau$.

L'équation (11) se réduisant à $z_{t}=y_{t}$, la solution du système (11), (12), (13) est encore immédiate :

$z_{t}=-\frac{\mathrm{T}_{t-(\tau / N)}-\mathrm{S}_{t-\tau}}{2}$

$$
+\sqrt{\left.\frac{T_{t-(\tau / N)}-S_{t-\tau}}{2}\right|^{2}+1}
$$

$x_{t}=z_{t}-\mathrm{S}_{t-\tau}$

Troisième cas : $x<0$.

Le calcul précédent doit être arrêté à la dernière valeur positive de $x$. Pour les valeurs suivantes $(x<0)$, il faut prendre le système complet faisant intervenir la perte de charge du clapet.

La résolution mathématiqne de ce système conduit à une équation du troisième degré. Plutôt que de résoudre cette équation, nous avons jugé préférable d'opérer par approximations en nous basant sur le fait que $y$ valiait d'une façon assez régulière (contrairement à $x$ et $z$ qui sont sujets à de brusques variations).

Pour chaque calcul, nous partons d'une valeur :

$$
y^{*}=2 y_{t-(\tau / \mathrm{N})}-y_{t-(2 \tau / \mathrm{N})}
$$

(extrapolation linéaire de $y$ ), valeur très voisine de la valeur $y_{t}$ cherchée. Les équations (11) et (12) par élimination de $z_{t}$ nous conduisent alors à une équation du second degré en $x_{t}$ dont la solution est :

$$
x^{\prime}=\frac{1-\sqrt{1+4 \lambda\left(\mathrm{S}_{\left.t-\tau-y^{*}\right)}\right.}}{2 \lambda}
$$

L'équation (13) donne ensuite une nouvelle valeur de $y$ :

$$
y^{\prime}=\frac{1}{\mathrm{~T}_{t-(\tau / N)}+x^{\prime}}
$$

Partant alors de $y^{\prime}$ nous caleulons un nouveau couple de valeurs $x^{\prime \prime} y^{\prime \prime}$, et ainsi de suite.

Connaissant ainsi $x_{t}$ et $y_{t}, z_{t}$ est donné immédiatement par l'équation (12) :

$$
z_{t}=x_{i}+\mathrm{S}_{t-z}
$$


Pratiquement, à la seconde itération, e'est-àdire au couple de valeurs $x^{\prime \prime} y^{\prime \prime}$, l'erreur résiduelle est négligeable et le calcul peut être arrêté là.

Graphiquement, il est facile de montrer l'excellente convergence de cette méthode. En effet, la détermination d'un point à l'instant $t$ se ramène à l'intersection d'une parabole (parabole de perte de charge du clapet rapportée à la droite Bergeron, ici droite à $45^{\circ}$ ) et d'une hyperbole (hyperbole du réservoir passant par le symétrique par rapport à l'axe des pressions du point à l'instant $t-[\tau / \mathrm{N}]$ ).

Or, en choisissant convenablement le fractionnement de la période, comme nous le verrons plus loin, l'hyperbole est très aplatie, alors que la parabole est très redressée (la pente de sa tangente est toujours supérieure à l'unité).

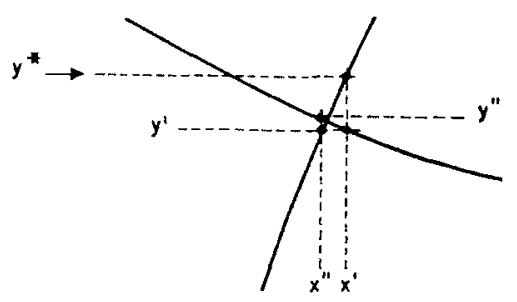

Fig. 5

L'itération correspond au trajet indiqué sur la figure 5, ce qui montre l'inutilité de poursuire très loin les calculs.

\section{Choix du fractionnement de la période.}

Il serait absurde de vouloir faire, dans certains cas, des calculs très précis, en ne cherchant que les points successifs séparés par un intervalle de temps égal à une période.

Inversement, un fractionnement excessif de la période conduirait à des calculs extrêmement longs autant qu'inutiles.

Il convient donc de ne tomber ni dans un excès ni dans lautre, mais bien au contraire de choisir une valeur judicieuse de N. Pour exposer les conditions de notre choix, il nous faut revenir en arrière.

Le premier point de l'épure (temps $\tau / N$ ) est défini par :

$$
\left\{\begin{array}{l}
z=y \\
z-x=z_{0}-x_{0} \\
\frac{1}{y}-x=\frac{1}{z_{0}}+x_{0}
\end{array}\right.
$$

ou :

$$
\frac{1}{z} z=\frac{1}{z_{0}}-z_{0}+2 x_{0}=\frac{1}{z_{0}}-z_{0}+2 \mathrm{~A} z_{1}
$$

Posons :

$$
z=k z_{0} \quad(k<1)
$$

Il vient :

$$
z_{0} ;:=\frac{1-k}{k[2 \mathrm{~A}-(1-k)]}
$$

La formule (7) nous donne alors $\mathrm{N}$ :

$$
\mathrm{N}=\frac{\mathrm{B}}{\mathrm{A}^{2}} \cdot \frac{1}{z_{\mathrm{i}^{2}}{ }^{2}}=\frac{\mathrm{B}}{\mathrm{A}^{2}} k \frac{[2 \mathrm{~A}-(1-k)]}{1-k}
$$

Comme critère, nous avons imposé a $z$, au temps $\tau / \mathrm{N}$, de ne pas différer de $z_{0}$ de plus de $10 \%$, d'où :

$$
k=0,9
$$

et par suite :

$$
\mathrm{N}=\mathbf{9} \frac{\mathrm{B}}{\mathrm{A}^{2}}(2 \mathrm{~A}-0,1)
$$

0,1 étant négligeable devant $2 \mathrm{~A}$, nous obtenons finalement :

$$
\mathrm{N}=18 \frac{\mathrm{B}}{\mathbf{A}}
$$

Cette formule donne une valeur non entière de $N$. Il faut, bien entendu, prendre pour $\mathrm{N}$ un nombre entier voisin de cette valeur.

Cette loi de fractionnement est purement conventionnelle mais conduit à des résultats très satisfaisants : calculs très précis sans être exagérément longs et convergence très rapide dans les itérations du calcul.

\section{Introduction de la perte de charge dans la conduite.}

Tout ce qui vient d'être dit, ainsi que les conclusions quantitatives qui seront données plus loin, suppose une perte de charge négligeable dans la conduite.

Or, il est possible, même facile, d'introduire une perte de charge sous la forme d'un diaphragme fictif placé dans la conduite au droit de la pompe. Ceci ne représente pas fidèlement le phénomène, mais donne des résultats bcaucoup plus proches de la réalité que ceux obtenus précédemment.

Soit $\beta w^{2}$ la perte de charge de la conduite et posons :

$$
\mu=\frac{\beta w_{10^{2}}}{\mathrm{H}_{9}} \sqrt{\frac{\mathrm{N}}{\mathrm{A}^{2} \mathrm{~B}}}
$$


les autres notations conservant la même signification.

Nous laissons au lecteur le soin de vérifier que les équations à résoudre sont maintenant les suivantes :

Premier cas : $x>0$ et $t<\tau$.

$$
\left\{\begin{array}{l}
z_{t}=y_{t} \\
z_{t}-\mu x_{t}^{2}-x_{t}=z_{0}-x_{0} \\
\frac{1}{y_{t}}-x_{t}=\mathrm{T}_{t-(\tau / \mathrm{N})}
\end{array}\right.
$$

DeuxiÈme cas : $x>0$ et $t>\tau$.

$$
\left\{\begin{array}{l}
z_{t}=y_{t} \\
z_{t}-\mu x_{t}^{2}-x_{t}=\mathrm{S}_{t-}= \\
\frac{1}{y_{t}}-x_{t}=\mathrm{T}_{t-(\tau / \mathrm{N})}
\end{array}\right.
$$

Troisitme GAS : $x<0$.

$$
\left\{\begin{array}{l}
z_{t}=y_{t}+\lambda x_{t}^{2} \\
z_{t}+\mu x_{t}^{2}-x_{t}=S_{t--} \\
\frac{1}{y_{t}} \cdots x_{t}=\mathrm{T}_{t-\{\tau / \mathrm{N}\}}
\end{array}\right.
$$

Il convient de donner à $S_{t}$ l'une des deux valeurs suivantes :

$\mathrm{S}_{t}=2 z_{0}-z_{t}+\mu x_{i}^{2}-x_{t} \quad$ pour $x_{t}>0$

$\mathrm{S}_{t}=2 z_{0}-z_{t}-\mu x_{t}^{2}-x_{t} \quad$ pour $x_{t}<0$

Dans chaque cas, la résolution du système peut être effectuée par itération, exactement comme il a été indiqué précédemment, plutôt que par résolution directe d'une équation du troisième degré.

\section{Détente adiabatique.}

Dans ce qui précède, comme du reste dans ce qui suit, nous avons supposé une détente isotherme dans le réservoir. Ceci constitue un cas particulier, et il n'est pas interdit de vouloir envisager le cas de l'adiabatisme. Les calculs précédents sont alors légèrement modifiés, mais conservent cependant leur simplicité.

La loi d'adiabatisme se traduit par la relation:

$$
H \mathrm{~V}^{\gamma}=\text { constante }
$$

Seule la troisième équation du système est modifiée et devient :

$$
\frac{1}{y_{t}^{1 / \gamma}} x_{t}=T_{t-(\tau / \mathrm{N})}=\frac{1}{\left(y_{t-(\tau / \mathrm{N})}\right)^{1 / \gamma}}+x_{t-(\tau / \mathrm{N})}
$$

en prenant maintenant :

$x_{0}=\mathrm{A} z_{0}$

$z_{0}=\left(\frac{\mathrm{B}}{\mathrm{A}^{2} \mathrm{~N}}\right)^{\gamma /(1+i)} \quad \lambda=\frac{\alpha w_{0}{ }^{2}}{\mathrm{H}_{0}} \cdot \frac{z_{0}}{x_{0}{ }^{2}}$

Les calcuIs, par la méthode d'itération, se poursuivent d'une manière analogue à ce qui a été fait précédemment, exception faite pour les valeurs successives de $y$ qui s'obtiennent par une expression de la forme :

$$
y_{t}=\frac{1}{\left(\mathbf{T}_{t-(\tau / \mathrm{N})}+x_{t}\right) \gamma}
$$

Pour l'adiabatisme, les calculs peuvent encore être confiés aux machines à cartes perforées et la perte de charge dans la canalisation peut également être introduite comme il a été indiqué dans le paragraphe précédent.

\section{DEUXIEME PARTIE}

\section{RESULTATS RELATIFS A LA DEPRESSION}

Nous avons appliqué systématiquement les calculs qui viennent d'être exposés à des couples de valeurs de $A$ variant de 0,5 à 4 et de $B$ variant de 0,05 à 1 .

Ceci nous a permis de constater que pour de faibles valeurs du rapport $B / A$ les résultats, en ce qui concerne la dépression, étaient très voisins de ceux obtenus par la théorie de l'oscillation en masse.

Par contre, dès que ce rapport n'est plus petit, les résultats peuvent diverger. 


\section{Dépression minimum.}

Pour la détermination de cette dépression minimum, il ne nous est pas possible de donner un abaque aussi complet que celui de MM. СомBES et Borot, puisque nous n'avons pas pris en compte la perte de charge dans la conduite.

Dans ce cas où la perte de charge est négligée, les résultats de l'oscillation en masse sont parfaitement vẻrifiés, l'erreur ne dépassant pas $1 \%$, tant que A est supérieur à l'unité.

Mais il n'en est plus de même pour $A<1$, et ceci se conçoit fort bien (fig. 6). En effet,

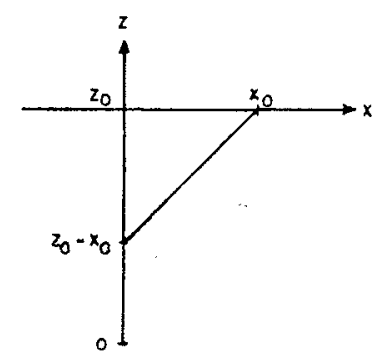

FIG. 6

$A<1$ entraine $x_{0}<z_{0}$ et la pression ne peut pas tomber au-dessous de $z_{0}-x_{0}$, d'où :

$$
\frac{\mathrm{H}}{\mathrm{H}_{0}}=\frac{z}{z_{0}}>\frac{z_{0}-x_{0}}{z_{0}}=1-\mathrm{A}
$$

Ceci donne le jeu de courbes représenté sur la figure 7 .

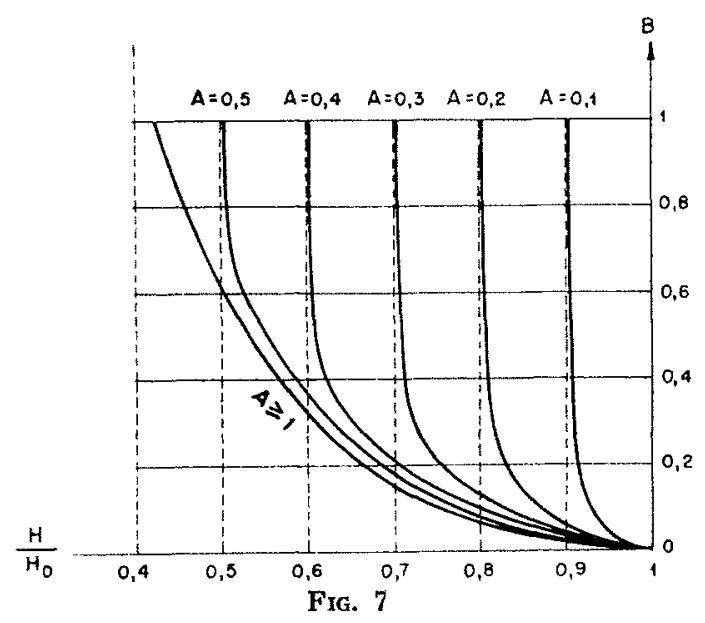

Ce cas constitue une exception, en matière de distribution d'eau, car, usuellement, A est supérieur à l'unité.

\section{Courbes des pressions minima.}

Le calcul donne à chaque instant la charge et la vitesse au droit du réservoir d'air. Mais le fait que la période ait été fractionnée en $\mathrm{N}$ parties permet d'obtenir immédiatement, de $\tau / \mathrm{N}$ en $\tau / \mathrm{N}$, les charges aux points de la conduite distants de la pompe d'un multiple quelconque de $\mathrm{L} / \mathrm{N}$.

Soit, en effet, à obtenir la charge à l'instant $t$ au point $M$ distant de la pompe de $n \times(\mathrm{L} / \mathrm{N})$ (fig. 8).
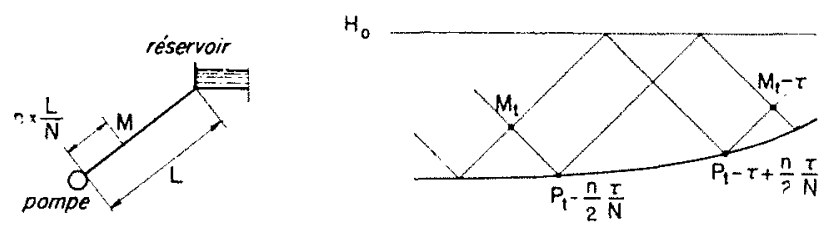

Fig. 8

Il est facile de vérifier que le point $M_{t}$ est à l'intersection des deux droites :

$$
\begin{gathered}
z-x=2 z_{0}-(z+x)_{t-\tau+(n / 2)(\tau / \mathrm{N})} \\
z+x=(z+x)_{t-(n / 2)(\tau / \mathrm{N})}
\end{gathered}
$$

d'où, par élimination de $x$ :

$$
\begin{gathered}
2\left(z_{0}-z\right)=(x+z)_{t-\tau+(n / 2)(\tau / N)} \\
-(x+z)_{t-(n / 2)(\tau / N)} \\
=S_{t-(n / 2)(\tau / N)}-S_{t-\tau+(n / 2)(\tau / N)}
\end{gathered}
$$

et :

$$
\frac{z}{z_{0}}=1-\frac{1}{2 z_{0}}\left(S_{t-(n / 2)(\tau / N)} \cdots S_{t}-\tau+(n / 2)(\tau / N)\right)
$$

Pour obtenir le minimum de la pression en

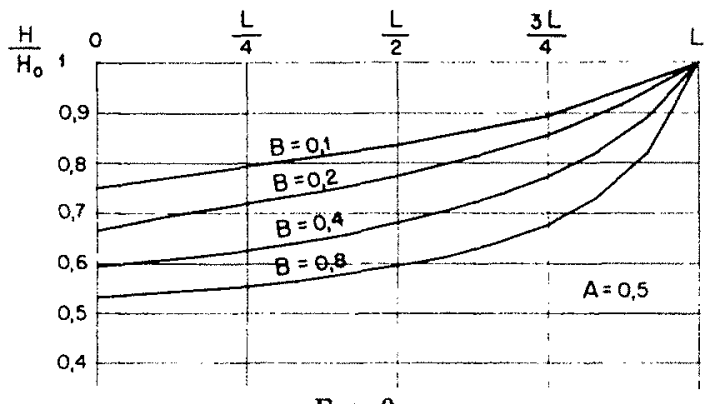

Frr. 9

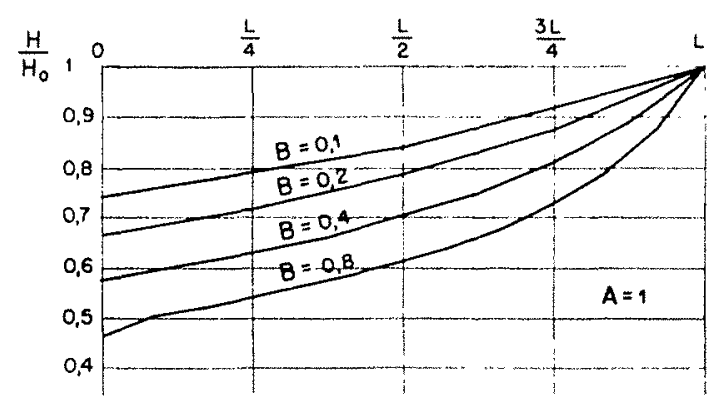

F1G. 10 


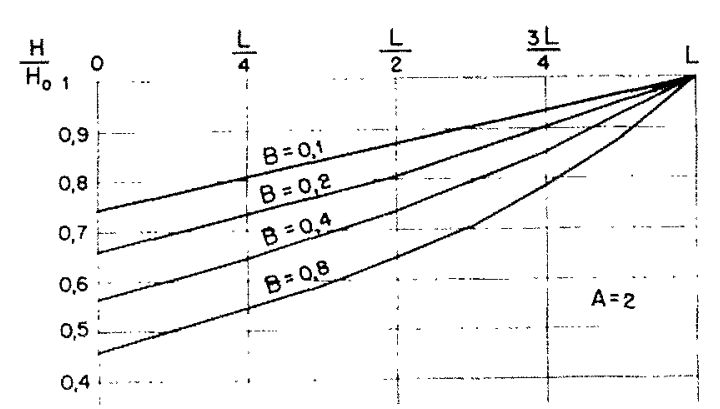

FIG. 11

chaque point $M$, il faut donc connaitte le maximum de la différence $\Delta S$ entre les quantités $S$ relatives a deux points $P^{P}$ distants, dans le temps de $[1-(n / N)] \tau$. Ces quantités $S$ étant connues, il est dès lor's facile de situer le minimum cherché.

Les trois familles de courbes suivantes (fig. 9, $10,11)$ donnent, pour $A=0,5 \quad A=1 \quad A=2$ et pour différentes valeurs de $B$, la pression minimum enregistrée en chaque point de la conduite.

La théorie de l'oscillation en masse donnerait. dans chaque cas, une droite et non une courbe. En d'autres termes, la concavité de ces courbes traduit la divergence avec la théorie de l'oscillation en masse.

Pratiquement, quand $\mathrm{B} / \mathrm{A}<1 / 10$, la courbe donnée par la théorie exacte de l'oscillation d'onde se confond avec la droite donnée par' la théoric approchéc de l'oscillation en masse.

\section{TROISIEME PARTIE}

\section{RÉSULTATS RELATIFS AUX SURPRESSIONS}

Dans cette troisième partie, où nous négligeons encore la perte de charge dans la canalisation, nous nous écartons totalement des hy-

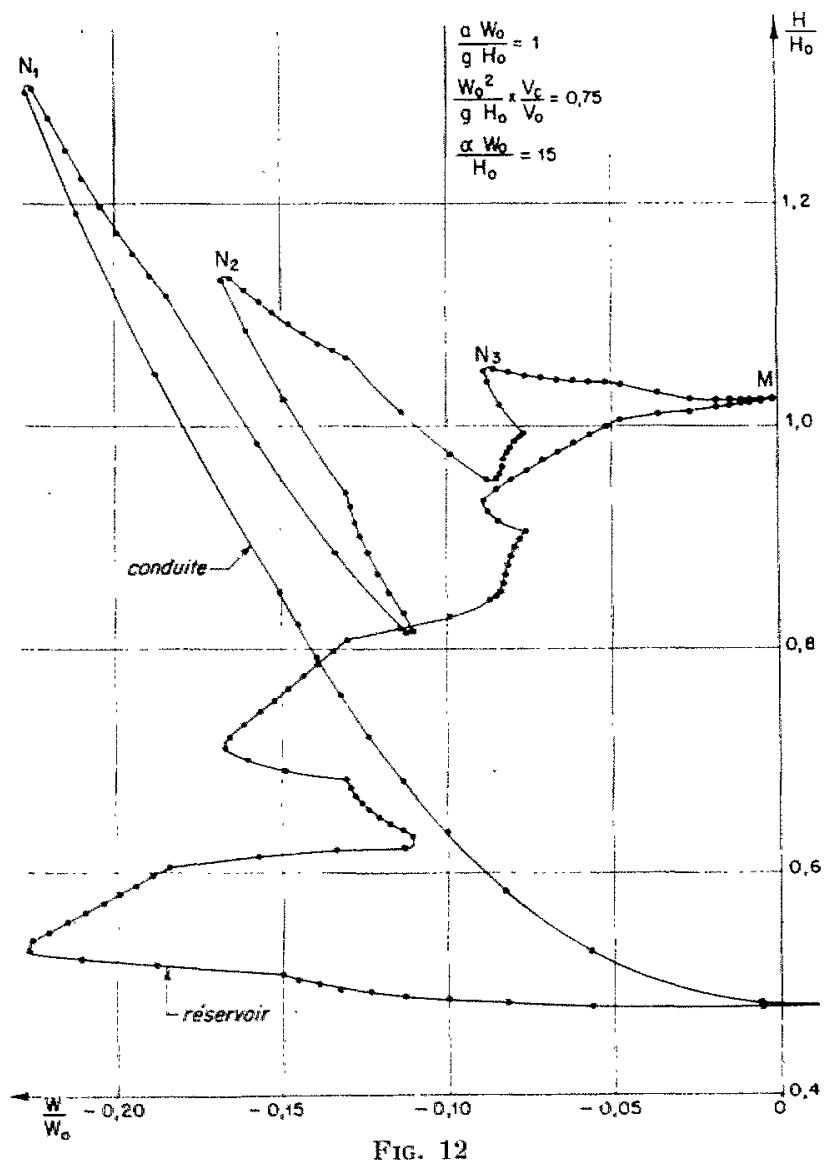

pothèses de l'oscillation en masse; l'épure (fig. 12. le montrera aisément.

Nous sommes en plein dans le domaine de l'oseillation d'ondes; en effet, la théorie de l'oseillation en masse ne permet pas d'obtenir les maxima et minima successifs observés dans la conduite. Il est possible de le démontrer mathématiquement, mais ceci nous entraînerait trop loin en sortant du cadre de cet exposé.

Le problème qui se pose est le suivant : connaissant A (quantité caractéristique de l'installalion) et $B$ (quantité définissant le volume du réservoir d'air), déterminer la perte de charge du clapet pour que la surpression dans la conduite soit minimum.

Ia théorie pure ne permet pas d'obtenir cet optimum, aussi faut-il opérer par tâtonnements; c'est ce que nous arons fait d'une façon systématique et ce que nous allons maintenant exposer.

\section{Recherche de l'optimum.}

Pour un couple de valeurs données de $A$ et $B$, il existe une infinité d'épures correspondant chacune à une valeur donnée de $\alpha$.

Examinons l'allure générale de ces épures dans la zone des vitesses négatives.

Quelle que soit la valeur de $\alpha$, la charge $y$ dans le réservoir est une fonction toujours croissante présentant un maximum $M$ pour $x=0$. En ce point, la charge dans la conduite est d'ailleurs égale à la charge dans le réservoir. Le 
maximum en question croît toujours lorsque $\%$ diminue.

Pour les valeurs élevées de o., la charge dans la conduite présente un ou plusieurs maxima $\mathrm{N}_{1} \mathrm{~N}_{2} \mathrm{~N}_{3}$ (fig. 12). Le plus important de ees

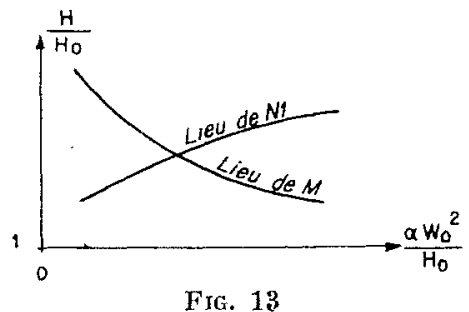

maxima est toujour's celui qui correspond a la vitesse la plus élevée en valeur absolue.

Lorsque $\alpha$ diminue, le maximum $\mathrm{N}_{1}$ diminue et finit même par disparaître.

L'optimum a lieu lorsque le maximum $M$ est égal au maximum $N_{1}$. On obtient alors une charge au-dessous de laquelle il est impossible de descendre $\left(^{*}\right)$.

La détermination de ce minimum parait inaccessible à la théorie pure. Nous avons donc effec-

(*) Pour les fortes valeurs du rapport $\mathrm{B} / \mathrm{A}(\mathrm{B} / \mathrm{A}>0$, approximativement), la courbe lieu du point $\mathrm{N}_{1}$ peut cesser d'être constamment croissante. L'optimum cherche correspond alors au minimum de cette courbe.

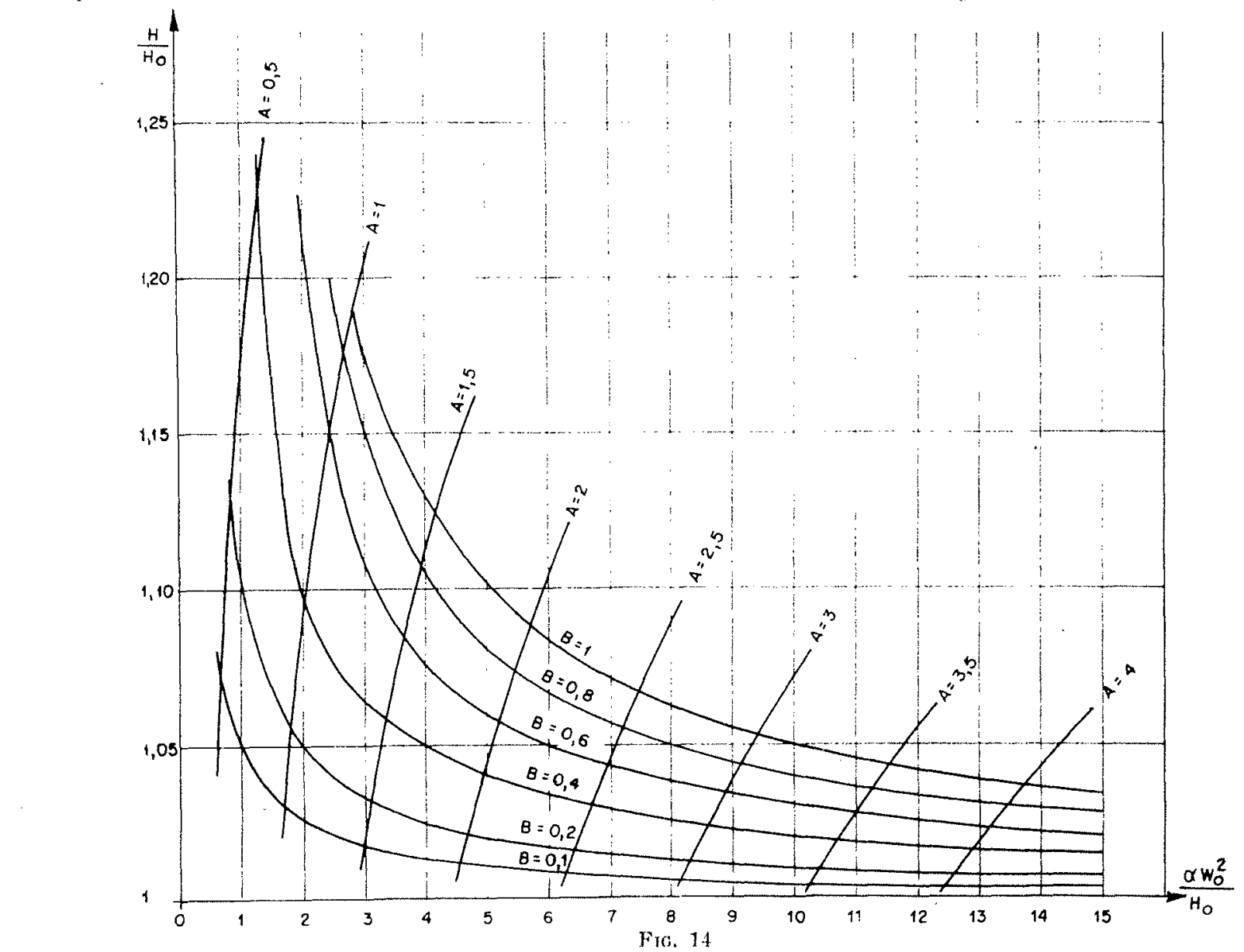

tué (à la machine) de nombreuses épures qui nous donnaient les valeurs respectives de $\mathrm{M}$ el de $N_{1}$ en fonction de $\alpha$. L'intersection des lieux de $\mathrm{M}$ et de $\mathrm{N}_{1}$ donne le a optimum et la valeur correspondanle de $\mathrm{H} / \mathrm{H}_{0}$ (fig. 13).

L'abaque (fig. 14) réunit les différents résultats que nous avons obtenus.

Pour les amateurs de mathématiques, zous pouvons signaler quelques formules que nous avons établies à partir do ces résullats numériques:

Courbes $\mathrm{A}=$ = constante :

$$
\frac{x v_{0}^{2}}{\mathrm{H}_{0}}\left(2-\frac{\mathrm{H}}{\mathrm{H}_{0}}\right)^{:}=\left(\frac{4 \mathrm{~A}}{3}\right)^{3 / 2}
$$

Courbes $B=$ constante:

$$
\frac{y_{0} \cdot w_{0}{ }^{2}}{\mathrm{H}_{0}}\left(\frac{\mathrm{H}}{\mathrm{H}_{0}}-1\right)=\frac{\mathrm{B}}{2}
$$

La première de ces formules cst très bien vérifice pour $A>1$. Elle donne encore de bons résultats jusqu'à $A=0,5$ à condition que $H / H_{0}$ soit inférieur à 1,25 .

La seconde formule est très bien vérifiée pour $\mathrm{H} / \mathrm{H}_{0}$ inférieur à 1,10 . Elle donne encore un bon ordre de grandeur jusqu’à 1,15. Il faut toutefois se garder de l'employer pour les très faibles valeurs de la perte de charge, car elle perd alors toute signification. 


\section{QUATRIEME PARTIE}

\section{UTILISATION PRATIQUE DES RÉSULTATS PRECEDENTS}

Généralement, le problème de la protection des installations élévatoires se présente aux ingénieurs de la façon suivante:

On connaît :

- la charge statique absolue $\mathrm{H}_{0}$,

- la vitesse en régime permanent $w_{0}$,

- la célérité des ondes $a$.

Ces trois données déterminent la quantité :

$$
\mathrm{A}=\frac{a w_{0}}{g \mathrm{H}_{0}}
$$

On connait en outre le profil en long de l'installation :

$1^{\circ}$ Si le profil en long présente une concavité accentuée (courbe $A$ de la fig. 2), il n'y a aucun risque de cavitation le long de la conduite et le volume du réservoir d'air peut être déterminé par l'une des courbes de la figure 7 (la courbe $A \geqslant 1$ étant identique à celle donnée par MM. Combes et Borot, au changement de notation près, pour le cas où la perte de charge est négligée);

$2^{*}$ Si le profil en long présente des points hauts où peut se produire la cavitation (courbe $B$ de la fig. 2), il convient alors de superposer ce profil en long à celle des figures 9,10 ou 11 qui correspond au cas étudić. Le volume du réservoir qu'il faut adopter sera déterminé par la valeur de :

$$
\mathrm{B}=\frac{w_{0}^{2}}{g \mathrm{H}_{0}} \cdot \frac{v_{c}}{v_{0}}
$$

indiquée sur la première des courbes qui ne coupe pas le profil en long.

Si cependant toutes ces courbes etaient coupées par le profil en long, la protection complète de l'installation, à partir de la station de pompage, nécessiterait un réservoir d'air de très grand volume, qu'il y aurait sans doute intérèt à remplacer par une protection localisée au point haut. Ceci étant un cas d'espèce, il ne nous est pas possible de donner une règle générale;

$3^{\circ}$ Dans ces conditions, la canalisation est protégée contre les risques de cavitation par le réservoir d'air dont le volume est fixé par la valeur de $B$ précédemment trouvée.

Pour limiter la surpression qui se manifeste dans la deuxième phase du phénomène, il faut placer entre le réservoir d'air et la conduite un organe dissymétrique générateur de perte de charge dans le sens conduite-réservoir d'air.

L'abaque de la figure 14 donne les conditions optima de fonctionnement, e'est-à-dire la surpression minimum $\mathrm{H} / \mathrm{H}_{0}$ et la perte de charge $\approx w_{0}{ }^{2} / \mathrm{H}_{0}$ de l'organe dissymétrique, en fonction des constantes A et B. Il suffit pour cela de lire les coordonnées du point d'intersection de la courbe $A=c^{\text {te }}$ et de la courbe $B=c^{\text {te }}$ correspondant à l'installation.

Si cet organe dissymétrique est un clapet percé, le diamètre $d$ du trou, rapporté au diamètre $\mathrm{D}$ de la canalisation principale, sera donné, en fonction du coefficient $\alpha$ de perte de charge, par la relation :

$$
\alpha=\frac{1}{2 g}\left[\frac{\mathrm{D}^{2}}{0,6 d^{2}}-1\right]^{2}
$$

$4^{\circ}$ Enfin, il se peut que, dans certains cas, le problème soit à prendre en sens inverse, la principale limitation imposée étant celle de la surpression à une valeur donnée $\mathrm{H} / \mathrm{H}_{0}$. Il suffit alors, sur l'abaque de la figure 14 , de prendre l'intersection de l'horizontale $\mathrm{H} / \mathrm{H}_{0}$ avec la courbe $\mathrm{A}=\mathrm{c}^{\text {te }}$. Ce point se trouve sur une courbe $B=c^{\text {te }}$ qui fixe le volume minimum du réservoir compatible avec les conditions imposées. D'autre part, l'abscisse de ce point donnera la perte de charge du clapet et donc le diamètre $d$ du trou.

A titre de contrôle, il faudra vérifier que, pour cette même valeur de $B$, la courbe des pressions minima est toujours au-dessus du profil en long. Si cette condition n'était pas vérifiée, il faudrait alors prendre un réservoir de volume supérieur.

Dans tous les cas, l'abaque précédent donnera la solution optimum :

- A volume égal du réservoir, détermination de la surpression minimum;

- A surpression égale, détermination du voIume minimum du réservoir d'air.

$5^{\circ}$ Nous signalerons, pour terminer, qu'il est possible d'avoir directement, et sans aucun calcul, le diamètre $d$ de l'orifice percé dans le clapet. Si nous nous reportons en effet à l'abaque de la figure 14, nous constatons que la perte de charge relative est sensiblement proportionnelle à $A$ :

$$
\frac{\alpha w_{0}^{2}}{\mathrm{H}_{0}}=k \mathrm{~A}=k \cdot \frac{a w_{0}}{g \mathbf{H}_{0}}
$$


$k$ étant un coefficient compris usuellement entre 2 et 3,5 .

Par suite, nous obtenons :

$$
\alpha=k \frac{a}{g} \cdot \frac{1}{w_{0}}
$$

Or, a, pour un rapport des diamètres $D / d$ suifisamment grand, a pour valeur :

$$
\alpha=\frac{1}{2 g}\left[\frac{1}{0,6}\left(\frac{\mathrm{D}}{d}\right)^{2}\right]^{2}=\frac{1}{0,72 g}\left(\frac{\mathrm{D}}{d}\right)^{4}
$$

d'où nous déduisons immédiatement :

$$
\frac{\mathrm{D}}{d}=\sqrt[ \pm]{0,72 k \cdot \frac{a}{w_{0}}}=k^{\prime} \sqrt[4]{\frac{a}{w_{0}}}
$$

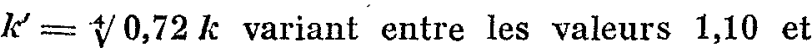
1,25 .

En valeur moyenne, nous pouvons adopter la formule :

$$
\frac{\mathrm{D}}{d}=1,2 \sqrt[4]{\frac{a}{w_{0}}}
$$

Dans le cas fréquent où $a=1.000 \mathrm{~m} / \mathrm{s}$ et $w_{0}=1 \mathrm{~m} / \mathrm{s}$, nous trouvons :

$$
\frac{\mathrm{D}}{d}=6,7
$$

ce qui donne l'ordre de grandeur du diamètre optimum de l'orifice.

EXEMPLE NUMÉRIQUE :

$$
\begin{array}{ll}
\mathrm{H}_{0}=60 \mathrm{~m} & a=1.200 \mathrm{~m} / \mathrm{s} \\
w_{0}=1 \mathrm{~m} / \mathrm{s} & g=10 \mathrm{~m} / \mathrm{s} / \mathrm{s}
\end{array}
$$

soit : $\mathrm{A}=2$,

et supposons que la nature du profil en long conduise, conformément à la figure 11 , à adopter un réservoir tel que $B=0,4$. Son volume serait par suite donné par :

$$
v_{0}=\frac{v_{o}}{240}
$$

Dans ces conditions, l'abaque de la figure 14 détermine le fonctionnement optimum :

$$
\frac{\alpha w_{0}^{2}}{\mathrm{H}_{0}}=4,95 \quad \frac{\mathrm{H}}{\mathrm{H}_{0}}=1,04
$$

d'où :

$$
\alpha w_{0}^{2}=297 \quad \mathrm{H}=62,40
$$

et :

$$
\frac{d}{\mathrm{D}}=\frac{1}{6,85}
$$

Cet exemple montre que, par un choix judicieux de l'évidement du clapet, la surpression peut être limitée à une très faible valeur, ici 2,40 mètres.

Notons que si nous avions pris un clapet plein, la surpression aurait été de 36 mètres. Sans clapet, elle aurait été de 60 mètres. La surpression devient le double de la valeur minimum si l'on prend un rapport des diamètres égal, soit à $1 / 6$, soit à $1 / 8$ environ, ce qui montre la rapidité de variation de la surpression en fonction du diamètre de l'évidement.

\section{Conclusion}

La méthode de calcul des épures Bergeron, au moyen des machines à cartes perforées, que nous venons d'exposer (sans toutefois entrer dans le détail de la programmation, qui ne peut intéresser que les spécialistes du calcul électronique) peut être féconde pour résoudre les problèmes qui exigeraient un grand nombre d'épures avant d'obtenir la meilleure solution.

Alors que le projeteur peut être découragé par ce nombre d'épures, la machine les exécute en se jouant.

A titre d'exemple, l'épure de la figure 12, comportant la détermination de 92 points successifs, a été achevée par la machine en une demi-heure; chaque point nécessite un passage de 36 cartes effectué en 20 secondes.

Si l'on veut bien examiner cette épure en détail, et nous n'employons ce mot ici que par routine puisque nous n'avons plus qu'une traduction graphique des résullats donnés par la machine, on constatera que la précision graphique, cu'elle aurait exigée d'un dessinateur employant la méthode classique, paraît hors de portée, sinon par une exécution à très grande échelle, d'ailleurs inhabituelle.

Dans le domaine des distributions d'eau, il semble que l'usage des machines électroniques ne soit pas limité aux calculs de coups de bélier : nous leur avons demandé notamment de résoudre les problèmes de réseaux maillés. Elles le font avec une rapidité qui paraît devoir surpasser, non seulement le calcul à la main, mais également le calcul par modèle. 